\title{
Self-reported hearing status and audiometric thresholds among college students using headphones
}

\begin{abstract}
Purpose: The study aims to investigate headphone listening habits of college-going students and for those using headphones, correlate self-reported hearing status with average audiometric hearing thresholds.

Method: Headphone listening habits and awareness of adverse effects of the same was profiled in college-going students using a questionnaire distributed through online platform. Hearing thresholds were then compared for those with and without self-report of hearing difficulty. 341 responses were obtained from students between 17 and 23 years of age. For the second part of the study, a convenience sample of 30 willing students from among these 341 was selected. Pure tone thresholds were obtained for various frequencies with a high frequency audiometer. PTA (average of 500, 1000, $2000 \mathrm{~Hz}$ ) and HFPTA (average of 4000, $6000,8000,10000 \mathrm{~Hz}$ ) were calculated for both the ears and compared for those with and without reported hearing difficulty.
\end{abstract}

Results: $78 \%$ students reported headphone usage for less than 3 hours per day, while $22 \%$ reported usage for more than 3 hours per day. $77 \%$ respondents were aware that listening to loud sounds can alter hearing sensitivity, but many (54.83\%) did not have awareness about the minimum safe hours of listening. There was a weak positive correlation between selfreported hearing difficulty and poor ear $\operatorname{HFPTA}(\mathrm{r}=0.2304)$.

Conclusion: Majority of students used insert earphones even after knowing the adverse effect of the same. There was a weak correlation found between the self-reported hearing problems and audiometric hearing thresholds.

Implication: More awareness is needed about the ill effects of headphone usage amongst the young teenage population. Proper counseling and management strategies are required for people who report difficulty in hearing.

Keywords: headphone listening, audiometric thresholds, high frequency pure tone average, college students, noise induced hearing loss
Volume I 3 Issue 3 - 202 I

\author{
Yash Shrimal,' Aparna Nandurkar ${ }^{2}$ \\ 'Audiologist \& Speech-Language Pathologist, India \\ ${ }^{2}$ Faculty, Department of Audiology, AYJNISHD(D), India \\ Correspondence: Yash Shrimal, Audiologist \& Speech- \\ Language Pathologist, Asha Speech and Hearing Clinic, New \\ Delhi, India, Tel +9173030 I2348, ORCID 0000-0003-0846-760I, \\ Email yash.shrimal26@gmail.com
}

Received: June 20, 2021 | Published: July 05, 2021
Abbreviations: PTA, pure tone average; HFPTA, high frequency pure tone average; NIHL, noise induced hearing loss; PMP, personal music player; SPL, sound pressure level; DPOAE, distortion product oto-acoustic emission

\section{Introduction}

Moore $^{1}$ describes noise as a sound that is unwanted and at an intensity with which it can interfere with verbal communication and may cause discomfort to the ears or reduction of hearing sensitivity, defined as hearing damage. In our daily surroundings, many sounds are present, some of which may be soothing and some may be perceived as noise leading to annoyance. Sounds which are at safe levels of loudness or shorter lengths of time are relatively safe and do not lead to hearing difficulties. However, those higher in intensity or occurring for longer durations may cause hearing problems progressively. Hearing function is affected by both long $^{2}$ and short-duration noise. The human ear, like any other body part can be damaged by overuse. The inner ear contains tiny hair cells that are gigantic in number. These tiny hair cells are susceptible to damage by loud sounds and prolonged exposure. ${ }^{3}$ Any exposure to noise of significant intensity and duration increases the risk of ear damage and causes permanent hearing damage, known as noise-induced hearing loss (NIHL). ${ }^{4}$ There can be a shift in hearing thresholds of the person after noise exposure. The shift in thresholds secondary to NIHL occurs in high frequency region or around $4000 \mathrm{~Hz}$ and higher making a dip/notch. The hearing loss starts and predominates in the frequencies of 3,4 , and $6 \mathrm{kHz}$ and eventually progresses to $8,2,1,0.5$, and $0.25 \mathrm{kHz} .{ }^{5}$

Recreational activity is a leading cause of NIHL and may include activities such as listening to loud music through headphones or insert earphones, target shooting, hunting, snowmobile riding or attending loud concerts. The noises produced during band activities, motor sports and loud noises in concert halls and nightclubs are known to have harmful effects on hearing. ${ }^{6}$

NIHL can occur in any age group and at any period of time due to exposure to loud sounds. Children, teenagers, adults and older people can be exposed to loud sounds and it can lead to NIHL. It is estimated that as many as 17 percent of teens (ages 12 to 19) have features of their hearing test suggestive of NIHL in one or both ears based on data from 2005-2006. ${ }^{7}$ The incidence of NIHL in children has been increasing day by day. For example, $40 \%$ of students between the ages of 16 and 25 years have audiological evidence of NIHL. ${ }^{8} 1 \%$ of children attending school were reported to have symptoms of NIHL in $1996 .{ }^{9}$

Listening to loud music or sound through earphones or headphones can lead to hearing loss. The World Health Organization ${ }^{10}$ has reported that loud music is the single biggest cause of preventable hearing loss. Approximately 1.1 billion young adults are at risk of hearing loss or 
loss in hearing sensitivity due to improper use of personal listening devices, including smartphones, and exposure to damaging levels of sound at loud noise entertainment surrounding such as discotheques, pubs and sport events. Studies have shown that exposure to music at high intensity and for longer periods of time is likely to induce many hearing symptoms, such as temporary threshold shift (TTS), tinnitus, hyperacusis, recruitment, distortion, or abnormal pitch perception. ${ }^{6,11,12}$ Studies on listening to music with headphones have indicated poorer hearing thresholds for adolescents and young adults who use headphones compared to those who do not use headphones. ${ }^{13}$

There is an increasing trend towards use of headphones for listening to music. Most people, especially youngsters, use them for considerable amounts of time, but do not realize the harmful effects that can be caused by prolonged usage of headphones on listening. Children and adolescents are also increasingly using portable digital audio players (DAPs), and some individuals, at maximum volume settings, with little knowledge of the risks this may pose to their hearing. ${ }^{14}$ Most school or college-going teenagers tend to use headphones for long hours without knowing the damage they are doing to their auditory mechanism. Adolescents and young adults (17-23 years) listen for 1.5 hours per day on average (ranging from 10 minutes to 4 hours), at a sound level of 73-79 dB A on average (ranging from 40 to $93 \mathrm{~dB} \mathrm{~A}$, depending on the device)..$^{15}$ Personal music players have very strong sound tones, and their maximum volume level can reach $78-136 \mathrm{~dB}{ }^{6}$ Individuals who listen to 15 minutes of music at $100 \mathrm{~dB}$ using personal music players may be exposed to the same level of loudness as industrial workers exposed to $85 \mathrm{~dB}$ in an 8 -hour day. ${ }^{16}$

The effect of headphone listening to music on hearing depends on several factors such as type of headphones ${ }^{17}$, number of hours of usage ${ }^{15}$, volume,$^{18}$ and type of music, to list a few. Numerous studies have indicated that further hearing exposure can be avoided using different strategies. This can be achieved by using different style of earphones and using an '80-90 rule'. ${ }^{18}$ Some devices show an alert notification if an individual exceeds $60 \%$ of volume as quoted by Portnuff. ${ }^{19}$

It is important to study how many of these young students report hearing related symptoms and how their self-perception of hearingrelated symptoms correlates with their hearing thresholds. The present study was taken up with the following aims:

a) To investigate headphone listening habits of college-going students,

b) For those using headphones, correlate self-reported hearing status with average audiometric hearing thresholds. The results of the study will help in creating awareness among college students about the detrimental effects of listening to loud music through headphones and in identifying hearing loss caused due to such exposure in the participants tested, serving as secondary prevention. The results will further aid in understanding the correlation between self-perception of hearing loss and clinically obtained hearing thresholds in college students who use headphones for listening to music, providing valuable information for counselling this population.

This study poses major questions such as: What are the headphone listening habits of college-going students? Is there awareness about recreational NIHL in college-going students using headphones? Are there any self-reported hearing related symptoms in college-going students using headphones? Are the pure tone thresholds different for students who report self-perception of hearing difficulty and for those who do not report self-perception of hearing difficulty? Is there a co-relation between self-reported hearing problems and clinical audiometric findings?

In order to achieve the aims of the present study the following objectives have been formulated:

a. To profile the headphone listening habits of college-going students

b. To study the awareness of college-going students about adverse effects of headphone listening on hearing sensitivity

c. To obtain information about the hearing related symptoms in college-going students using headphones.

d. To obtain hearing thresholds of college-going students who use headphones for listening and compare hearing thresholds of college-going students for those with self-report of hearing difficulty and those without self-report of hearing difficulty.

e. To obtain correlation between self-reported hearing status and average hearing thresholds.

\section{Materials and methods}

Approval was obtained from the Institutional Ethics Committee and all procedures were as per the approved protocols. The study was divided into two parts. In Part 1, proforma-based survey to assess headphone listening habits was distributed online amongst many students and colleges of which 393 responses were received. In Part 2, 30 participants from Part 1 of the study were seen for audiological assessment to obtain their hearing thresholds.

\section{Participants for part I - survey using online proforma}

Both male and female students attending degree colleges in Mumbai and suburbs and comfortable with English language were included as participants for this phase. A survey form was framed keeping in mind that the participants are college students who may not be well versed with scientific terms. The questions were framed in simple layman terms and the number of questions were kept to a minimum so that the participant would fill the proforma with full interest without consuming lot of time to increase the chances of obtaining complete and valid responses. The tool had both open ended and close ended questions. The questions included
a. Demographic data,
b. History,
c. Device use,
d. Hearing related symptoms and
e. Awareness about effect of headphone listening on hearing.

Content validity of the developed proforma was established by five audiologists with more than 10 years of clinical experience. The suggestions were incorporated, and changes were made as suggested by the validators. The final proforma was made using 'Google Forms' online and link was generated for the same to be forwarded to participants. The Google form was distributed on mobile phones via E-mail, text messages or social media to participants fulfilling the inclusion criteria. 393 students responded to the survey; however, some did not fulfil the inclusion criteria and 36 returned incomplete proforma. Hence, a total of 341 participants were finally included in this part of the study. The details of these 341 respondents are shown in Table 1 below. 
Table I Respondents for the online survey

\begin{tabular}{lllllllll}
\hline & $\begin{array}{l}\text { Number of } \\
\text { participants }\end{array}$ & $\begin{array}{l}\text { Age Range } \\
\text { (years) }\end{array}$ & $\begin{array}{l}\text { Mean age } \\
\text { (years) }\end{array}$ & \multicolumn{2}{l}{ Hours of usage per day } & \multicolumn{2}{l}{ Years of usage } & \\
\hline & & & & $>3$ & $<3$ & $<2$ & $02-$ May & $>5$ \\
Male & 138 & $17-23$ & 19.81 & 39 & 99 & 24 & 63 & 51 \\
Female & 202 & $17-23$ & 19.42 & 36 & 166 & 53 & 96 & 53 \\
Other & I & 18 & 18 & 0 & 1 & 1 & 0 & 0 \\
Total & 341 & $17-23$ & 19.58 & 75 & 266 & 78 & 159 & 104 \\
\hline
\end{tabular}

\section{Participants for part 2 - pure tone audiometry}

For the second part of the study, a convenience sample of 30 willing participants ( 23 males, 7 females) from among these 341 was included such that they had more than 3 hours per day usage of headphones and exposure to headphone use for minimum 2 years. Participants may or may not have reported hearing difficulty in the survey. Students reporting presence of middle ear pathology, any neurological impairment, history of ototoxicity or sudden unilateral or bilateral hearing loss were excluded.

\section{Procedure}

For the first part of the study, the developed Google link was distributed through e-mails, WhatsApp and other social media to various students from many degree colleges in Mumbai and suburbs. The link had the instructions to answer all questions to complete the proforma. Participants were also allowed to not disclose their identity (name) if they wished to do so. As the data for the first part of the study was being collected online, simultaneously the second part of study was initiated. Students who were willing to visit the clinic at their convenience were asked to report for audiological evaluation. After obtaining their written informed consent, detailed history was obtained on a case record form and pure tone audiometry was conducted in a standard sound treated room as per ANSI guidelines. Pure tone thresholds were obtained using calibrated high frequency audiometer Resonance r37a Clinical with Sennheiser HDA 280 calibrated headphones for the frequencies $250 \mathrm{~Hz}, 500 \mathrm{~Hz}, 1 \mathrm{kHz}, 2$ $\mathrm{kHz}, 4 \mathrm{kHz}, 6 \mathrm{kHz}, 8 \mathrm{kHz}, 10 \mathrm{kHz}$ and $12.5 \mathrm{kHz}$. Prior to audiometry, otoscopy was performed using Welch-Allyn otoscope to rule out any symptoms associated with outer or middle ear. The obtained thresholds were recorded on the case record sheet and pure tone averages were obtained as follows:

a. PTA right - average of pure tone thresholds of right ear at frequencies $500 \mathrm{~Hz}, 1 \mathrm{kHz}$ and $2 \mathrm{kHz}$;

b. PTA left - average of pure tone thresholds of left ear at frequencies $500 \mathrm{~Hz}, 1 \mathrm{kHz}$ and $2 \mathrm{kHz}$;

c. HFPTA right - average of pure tone thresholds of right ear at frequencies $4 \mathrm{kHz}, 6 \mathrm{kHz}, 8 \mathrm{kHz}$ and $10 \mathrm{kHz}$

d. HFPTA left - average of pure tone thresholds of right ear at frequencies $4 \mathrm{kHz}, 6 \mathrm{kHz}, 8 \mathrm{kHz}$ and $10 \mathrm{kHz}$.

For the purpose of analysis of this data to fulfil the objectives, the 30 participants in this part of the study were divided into two groups - those who reported to perceive hearing difficulties $(\mathrm{N}=12)$ and those who did not perceive hearing difficulties $(\mathrm{N}=18)$. Self-perceived hearing difficulty was determined on the basis of two questions from the survey proforma.
1. Do you have difficulty in hearing?

2. Do you have difficulty in understanding speech of others? If the answer to any one of these questions was "yes", the respondent was considered to self-perceive a hearing problem (refer) and if both the questions were answered as "no", the respondent was considered to have no self-reported hearing difficulty (pass).

The responses obtained on each of the five categories of the questionnaire were converted into MS Excel sheets and percentage of respondents providing the various responses to each question was calculated. For the second part of the study, pure tone averages (PTA right and PTA left) and high frequency pure tone averages (HFPTA right and HFPTA left) were calculated for both the groups i.e., those who did not perceive hearing related problems and those who perceived to have hearing related problems. Kolmogorov-Smirnov Test of Normality was performed to check if the data for PTA right, PTA left, HFPTA right and HFPTA left was normally distributed. Kolmogorov-Smirnov Test of normality results revealed that the data for PTA right and PTA left ear were normally distributed while data for HFPTA right and HFPTA left was not normally distributed. Based on the results of the test of normality, comparison of the two groups for PTA right and PTA left was done using unpaired $t$ test, while Mann Whitney $\mathrm{U}$ test was performed to compare the data of the two groups for HF PTA right and HF PTA left. To obtain correlation between self-reported hearing status and average high frequency hearing thresholds, the data was analysed in the following manner. The four questions pertaining to self-perception of hearing difficulty (i.e. do you have difficulty in hearing, do you have difficulty in understanding speech of others, do you often ask for repetition, do you hear ringing or hissing sound inside ear in quiet?) were answered as YES or NO. Scoring was done in such a way that "yes" was scored as 1 point and "no" was scored as 0 point. A total score of 4 could thus be obtained, wherein higher score is indicative of greater perceived problem. This score was correlated with the HFPTA of the ear which was poorer of the two ears using the Pearson correlation coefficient value ' $r$ '.

\section{Results}

The results of the study are presented below with reference to the objectives listed in introduction.

\section{A. Profile of headphone listening habits of college- going students}

i. Type of listening devices and transducers used: 296 of the 341 respondents $(87 \%)$ used cell phones as their personal listening devices (PLDs) for listening to music. 26 participants (7\%) used laptops, $10(3 \%)$ used iPods and only $9(3 \%)$ respondents reported to use Personal Music Players. Majority of the respondents ( 272 i.e. $80 \%$ ) used insert earphones as their 
transducer followed by headphones (30 participants), portable speakers (26 participants) and the least used transducer was Loudspeaker (13 participants).

ii. Volume of the device: The volume levels reportedly used by the different number of participants are depicted in Figure 1. As seen from Figure 1, participants reported varied volume levels at which they usually listen to music. Volume levels 8,7 and 6 were the most widely used, while very low volume levels such as level 2 or 3 were reported by very few participants. When participants were asked if loud sounds are intolerable to them, 63 of them found them to be intolerable and 179 reported this to happen sometimes. 41 participants found male voice to be better perceived than female and most of them (i.e., 261) had not noticed such difference of perception in male and female voice.

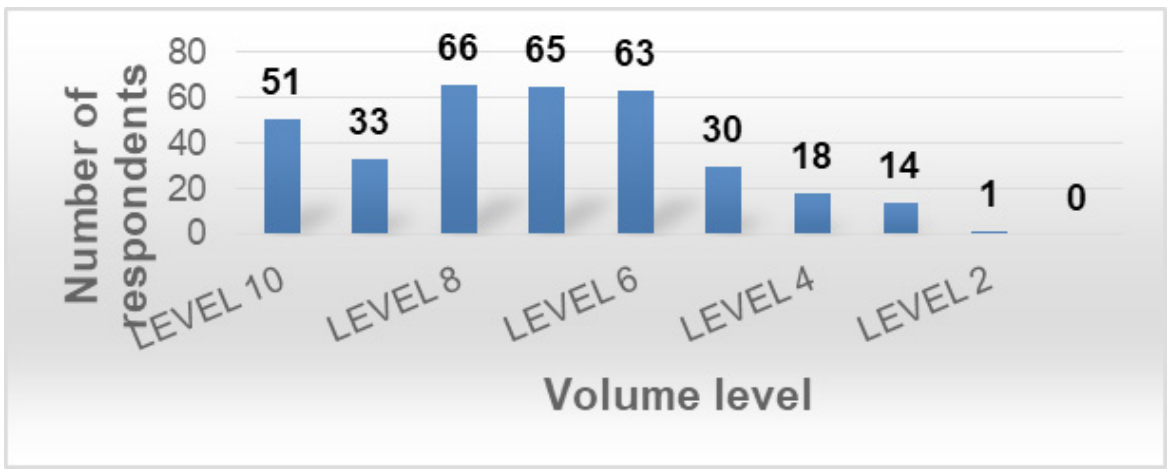

Figure I Number of participants reporting the different volume levels while listening to music.

iii. Number of hours of usage: $78 \%$ of the respondents used headphones for less than 3 hours per day while $22 \%$ used them for more than 3 hours per day. 104 participants (30\%) reported to have been using headphones or PLDs since more than 5 years and $78(23 \%)$ reported to have been using them for less than 2 years.

iv. Activities during which headphones are used: Respondents were asked to list the activities that involve more headphone usage during their daily routine. The most common situations during which most respondents used headphones were in public places, while commuting and while working out. Type of activity also had effect on the volume level at which respondents listened, because some activities involved higher background noise. The data showing percentage of respondents using headphones during various activities is depicted in Figure 2.

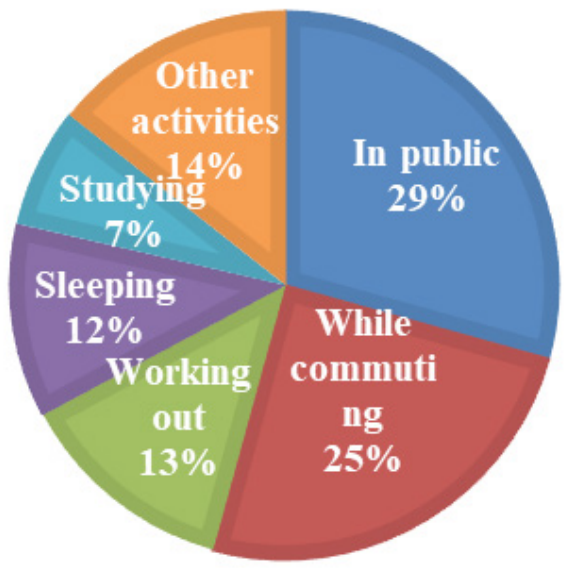

Figure 2 Percentage of respondents using headphones during various activities.

v. Exposure to other noisy activities: When participants were asked about their involvement in any other recreational activity which involved high intensity sounds, only $18 \%$ reported to be involved in such activity (regular clubbing, discos, use of loud firecrackers, noisy neighbourhood), while others were just exposed to sound from their Personal Listening Devices or Laptops.

\section{B. Awareness of college-going students about adverse effects of headphone listening on hearing sensitivity}

Participants also responded to questions addressing their awareness about the hazardous effects of exposure to loud music and excessive headphone usage and their awareness about how the hazardous effects can be prevented. 261 of the 341 respondents (77\%) were aware that listening to loud sounds can alter hearing sensitivity but many $(54.83 \%)$ did not have awareness about the minimum safe hours of listening and most $(64.80 \%)$ were not aware about ways to prevent effects related to exposure to loud sounds.

\section{Hearing related symptoms in college-going students using headphones}

Information about any past or present hearing related symptoms was also obtained from the respondents. $70 \%$ of the respondents had no history of any middle ear infection. $30 \%$ had some middle ear related issue but only 6 out of 341 subjects had a history of ear discharge. Only $14 \%$ respondents reported to have any neurological issues while $86 \%$ had no history of any such issues. Among participants who reported to have history of neurological problems, migraine was the most commonly occurring problem. With reference to systemic illnesses and other infections, 33 out of the 341 participants reported to have had Malaria/dengue in their past. 255 participants had no history of any major illness, 8 had problems related to blood pressure. Seven participants also reported to have hearing related problems or hearing loss in their family. Figure 3 shows the number of respondents with ear-related and neurological problems.

Participants were also asked about hearing-related symptoms that they may be experiencing, the data for which is shown in Figure 4. 203 of them reported to have no such difficulty and the remaining subjects reported to have some or the other problems in hearing. Participants were asked if they ever needed to ask for repetition asking 'what', most of them (i.e. 274) said they need to ask for repetition sometimes 
and 47 reported they never did, and few (i.e.20) reported that they always do. 108 participants reported that people around them have stated that they can hear their music outside of the headphones. 44 respondents also reported to have problem in speech perception of others. Tinnitus was also reported by 80 out of 341 participants.

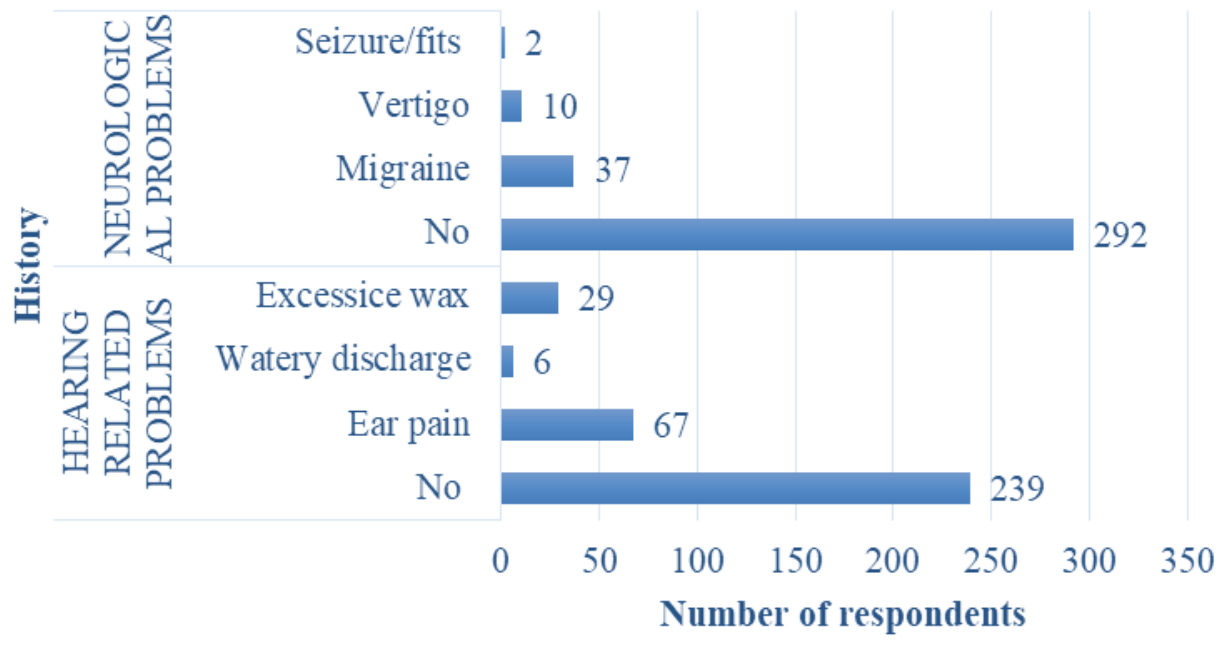

Figure 3 Number of respondents with history of ear-related and neurological problems.

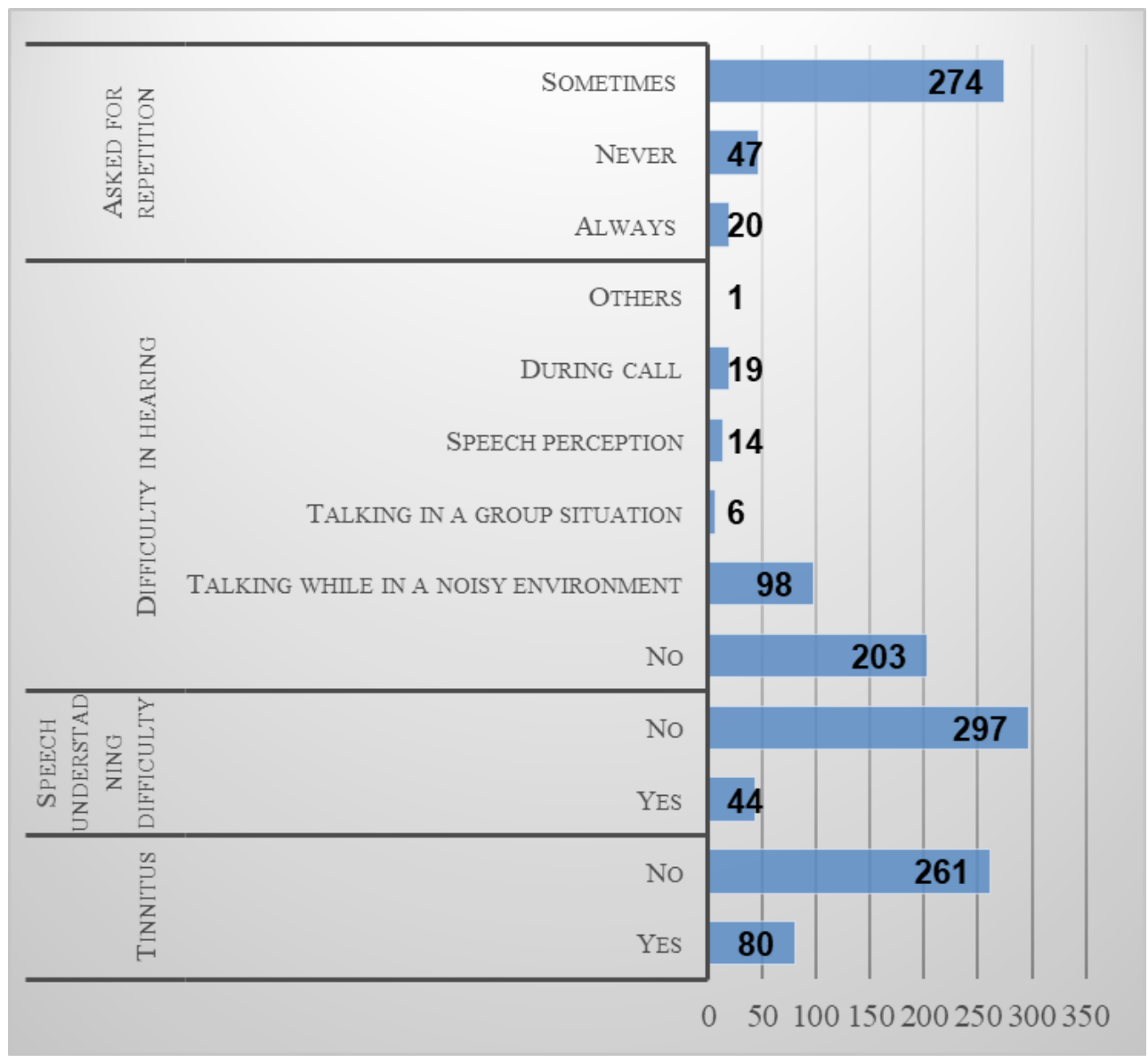

Figure 4 Number of respondents with different hearing related symptoms.

D. Hearing thresholds of college-going students who use headphones for listening

Out of the 341 respondents, 30 were taken up for hearing testing using pure tone audiometry. Two pure tone averages were obtained for each ear of the 30 participants - average of $500 \mathrm{~Hz}, 1000 \mathrm{~Hz}$ and 2000
$\mathrm{Hz}$ (PTA) and average of $4000 \mathrm{~Hz}, 6000 \mathrm{~Hz}, 8000 \mathrm{~Hz}$ and $10000 \mathrm{~Hz}$ (HF PTA). The 30 participants were divided into 2 groups -12 who reported to perceive hearing difficulties and 18 who did not report any hearing difficulties. The average PTA and HF PTA values with SD and percentiles for the two groups of participants are shown in Table 2. 
Table 2 Descriptive statistics for PTA and HF PTA for the two groups

\begin{tabular}{llllll}
\hline Self-reported & Statistics & $\begin{array}{l}\text { PTA Right } \\
(\mathbf{d B H L})\end{array}$ & $\begin{array}{l}\text { PTA Left } \\
(\mathbf{d B H L})\end{array}$ & $\begin{array}{l}\text { HFPTA Right } \\
(\mathbf{d B H L})\end{array}$ & $\begin{array}{l}\text { HFPTA Left } \\
(\mathbf{d B H L})\end{array}$ \\
\hline Yes & Mean & 15.93333 & 14.425 & 18.8375 & 10.7291 \\
& SD & 6.101167 & 3.715114 & 14.94463 & 5.601804 \\
& Range & Oct-30 & Oct-20 & $7.5-52.3$ & $2.5-22.5$ \\
& Median & 15.8 & 14.45 & 15 & 9.375 \\
& 25th percentile & 11.6 & 10.4 & 8.75 & 7.8125 \\
& 75th percentile & 19.15 & 17.875 & 20.3125 & 15 \\
& Mean & 16.91111 & 17.28333 & 17.7083 & 18.6555 \\
& SD & 4.249275 & 6.790846 & 12.0221 & 15.84016 \\
& Range & Oct-25 & $10-38.3$ & $10-63.75$ & $8.75-77.5$ \\
& Median & 16.6 & 15.8 & 14.37 & 13.84016 \\
& 25th percentile & 13.3 & 11.6 & 12.5 & 11.25 \\
& 75th percentile & 20.4 & 20.4 & 17.5 & 19.0625 \\
\hline
\end{tabular}

As seen from Table 2, the mean PTA right for those with no selfperceived hearing difficulty (16.91 dBHL) is higher than that for participants with self-reported hearing difficulty $(15.93 \mathrm{dBHL})$. The mean PTA left for the participants with no self-reported difficulty in hearing is also higher (17.28 dBHL) than that for participants with self-reported hearing difficulty (14.42 dBHL). The group with selfreported hearing difficulty had greater SD for both PTA right and left than the group with no self-reported hearing difficulty. Unpaired t-test (PTA Right $-\mathrm{t}=0.5187, \mathrm{p}>0.05$; PTA Left $-\mathrm{t}=1.3267, \mathrm{p}>0.05$ ) indicates that there is no significant difference between the average PTA right and PTA left of participants with and without self-reported hearing difficulty.

As seen from Table 2, the mean HF PTA right for the participants with self-reported difficulty in hearing was higher (18.837 dBHL) than that for the participants with no self-reported hearing difficulty (17.7083 dBHL). The group with self-reported hearing difficulty had greater SD value than the group with no self-reported hearing difficulty. Mann-Whitney $U$ test $(Z=0.52917 ; p=0.59612)$ indicates that there is no significant difference between the average HF PTA right of participants with and without self-reported hearing difficulty. The mean HF PTA left for those with no self-perceived hearing difficulty is higher (18.65 dBHL) than that for participants with selfreported hearing difficulty (10.7291 dBHL). The group with no selfreported hearing difficulty had greater SD value then the group with self-reported hearing difficulty. Mann-Whitney $U$ test $(Z=2.2225$; $\mathrm{p}=0.02642$ ) indicates that there is significant difference between the average HF PTA left of participants with and without self-reported hearing difficulty.

\section{E. Correlation between self-reported hearing status and average hearing thresholds}

For the 30 participants in part 2 of the study, the self-reported hearing difficulty score was obtained as described above based on the responses provided on the proforma. The mean score was 1.56 ( $\mathrm{SD}=0.989$, Range 0-4). The HFPTA of the poorer ear for each of the 30 participants was considered to obtain the mean HF PTA (19.95 dBHL, SD=14.49, Range 7.5-77.5). The correlation coefficient between the self-reported hearing difficulty score and poor ear HF PTA was found to be $r=0.2304$, indicating a weak positive correlation between the two measures.

\section{Discussion}

In this advancing world people have started to rely on technology throughout the day, especially teenagers who are never seen without their phones or laptops, always almost plugged in with headphones/ earphones for recreational purpose. The current study aimed at exploring the headphone listening habits of college-going students, studying their awareness about ill effects of excessive headphone usage and correlating self-reports of hearing difficulty and audiometric thresholds. The results obtained from the collected data are discussed here for the various objectives of the study.

\section{Profile of headphone listening habits of college-going students}

The results indicate that most of the respondents used cell phones as their personal music players; same as reported by Gupta et $\mathrm{a}^{20}$ and $80 \%$ of the respondents used insert earphones which was also seen in other studies as that by $\mathrm{McNeil}{ }^{21}$ whose study reported most of the participants using insert earphones as their transducers but iPod was the most commonly used personal music player amongst their sample. Use of Personal Music Player was found to be minimum in the present study; probably due to availability, cost and trend among peers. Results of the present study also indicate that college students usually tend to listen to music at high volume levels i.e. $70-80 \%$ of the maximum volume. This was also a common finding reported in other studies. ${ }^{20,22}$ Herrera et al..$^{23}$ reported that $37.40 \%$ of their participants used high volume when listening to music, $34.35 \%$ at medium volume and $16.03 \%$ at extreme high volume and only about $3.05 \%$ at low volume. Findings of these past studies as well as the present study imply that most of the young population have a habit of listening at high volume levels. Some studies ${ }^{22}$ also state that men usually tend to keep the volume higher. However, comparison between male and female participants was not done in the present study. Respondents of the present study were also asked if they perceive male voices better 
than female voices in order to get an indication of any high frequency hearing loss that they might have developed due to noise exposure. However, the results indicated that most of them haven't noticed such a difference, but 41 respondents noticed that they perceive male voice better then female voice. In the present sample, $78 \%$ respondents used headphones for less than 3 hours per day, which is in agreement with the study by Gupta et al. ${ }^{20}$ wherein $77.4 \%$ participants listened to music for an hour or less than an hour. Current findings also uphold reports by Berg et al. ${ }^{24}$ and Herrera et al. ${ }^{23}$ Similar results are reported by Torre ${ }^{22}$ who studied a very large sample (i.e., 1016) and more than $50 \%$ of the sample reported to use personal music players between 1-3 hours per day; and men usually used personal music players for longer duration than females. Male-female comparison was not made in the present study. Present results about duration of usage are contradictory to those of Shah, ${ }^{25}$ who found most of their sample to report more than 4 hours of usage per day and also reported that females listened to music at high levels for longer durations than men. $30 \%$ of the respondents in the present study reported to have been using headphones for more than 5 years and only about $23 \%$ reported to be using headphones less than 2 years, others had exposure between 2 to 5 years. Considering that these participants are teenagers, already having headphone usage at loud volumes for long durations for many years, and likely to continue to indulge in similar behaviours for several more years, their risk for NIHL increase significantly. This necessitates provision of appropriate counselling regarding ill effects of exposure to loud music for prolonged durations.

Headphone usage does not just occur at home sitting in quiet environment and in a teenager's life when there is a lot of commute and other activities, listening to music goes hand in hand. Study sample reported to be using headphones commonly in public places, while commuting and while working out. Similar results were obtained from various studies which involved questions regarding the habits and usage of headphones and personal music players. ${ }^{21,22,26,27}$ Such usage puts them at risk of using listening to music at higher volumes and louder levels as reported by Hodgetts et al. ${ }^{28}$ wherein Preferred Listening Levels (PLL) were higher in street noise than in multi talker babble and both were higher when compared to PLL in quiet Noise exposure is not only through headphones in a teenager's daily living. People tend to explore and indulge in many other recreational activities which involve loud sounds or noises which could also increase the total amount of daily noise exposure for the individual. In the present sample only $18 \%$ of the respondents reported to be indulged in such activities regularly. $10 \%$ of respondents reported to spend lots of time in noisy environments e.g. railway station, traffic, main roads, music events etc. $4 \%$ of the respondents were involved in regular clubbing or going to discotheques. $1 \%$ of respondents reported to be involved in loud gun fire or firecracker activities. Exposure to these activities might have an impact on hearing sensitivity/thresholds. Keppler et al. ${ }^{29}$ reported temporary tinnitus in $86 \%$ of respondents and also compared hearing thresholds of groups who were exposed to low, intermediate, and high recreational noise exposure. The results showed no significant difference in thresholds between the groups, though long-term exposure may lead to decrease in hearing sensitivity in future so long-term assessment may be required.

\section{Awareness of college-going students about adverse effects of headphone listening on hearing sensitivity}

It is of utmost importance to be aware of the safety levels and hours of usage of personal music players, as one can know to what limit it should be used daily so that there is no harm to hearing mechanism. 261 of the 341 respondents (77\%) were aware that listening to loud sounds can alter hearing sensitivity but many (54.83\%) did not have awareness about the minimum safe hours of listening and most $(64.80 \%)$ were not aware about ways to prevent effects related to exposure to loud sounds. Study by Shah et al. ${ }^{25}$ also included a question regarding awareness of NIHL and concerns regarding its effect. Most of the participants were at least slightly concerned about hearing loss with aging ( $15 \%$ were "not concerned"). Majority participants were also concerned with hearing loss related to use of their devices ( $24 \%$ were "not concerned"). Another study by Berg L et $\mathrm{al}^{24}$, compared knowledge about safety levels amongst males and females, a greater number of female students chose right answer to safe usage of PLD and safe listening per day than men. Herrera et al..$^{23}$ also studied awareness and concerns regarding hearing health and found that $13.74 \%$ were concerned about hearing loss via the use of personal music players, $36.64 \%$ were more or less concerned, $12.96 \%$ were very concerned, $23.66 \%$ were not very concerned and $11.45 \%$ were not concerned at all. Same results were seen in other studies. ${ }^{20}$

\section{Information about the hearing related symptoms in college-going students using headphones}

As this study focuses on effects of headphone on hearing, it was important to exclude those subjects from the sample who already might have a hearing loss or may develop hearing related problems due to causes like middle ear infection, genetic hearing loss, neurological problems, etc. This was important for part 2 of the study. $70 \%$ of the respondents had no history of any middle ear infection. $30 \%$ had some middle ear related issue but only 6 out of 341 respondents had a history of ear discharge. Similar findings were reported by Torre ${ }^{22}$ and Kumar. ${ }^{15}$ Båsjö et al..$^{30}$ also reported $81.1 \%$ (331/408) had normal middle-ear function in both ears. The prevalence of bilateral middleear abnormalities was $7.8 \%(32 / 408)$, and $11.0 \%(45 / 408)$ of the children had unilateral middle-ear abnormalities. This study also showed the relationship between middle ear pathology and PTA. It was reported that sample who had history of middle ear pathology showed poorer thresholds. To better understand if the respondents self-perceived any hearing related symptoms, direct questions and some indirect questions were framed to have an understanding about the perceived hearing symptoms of the respondents. When respondents were asked if they perceive to have hearing difficulty, 203 subjects reported to have no such difficulty and the remaining subjects reported to have some or the other problems in hearing properly. Similar results were reported by Widen et al. ${ }^{26}$ where out of 50 adolescents, $30(60 \%)$ reported having no hearing problems and $8(16 \%)$ reported poor hearing. Indirect question i.e. if they ever needed to ask for repetition asking 'what', revealed that most of them (i.e. 274) need to ask for repetition sometimes, 47 reported they never did, and few (i.e.20) reported that they always do. Respondents were also asked if people around them state or report that they can hear the loud music outside from their earphones, 108 respondents agreed that they have been pointed for the same. In such say we can make the respondents realise that even unconsciously they are listening to loud music throughout the day and without feeling that there might be problem in their hearing.

Tinnitus is one of the most common symptoms seen in persons who might have NIHL or long duration music exposure.13,6 Tinnitus was also reported in 80 subjects out of 341 in our sample, which is just $23.4 \%$. Various studies reported tinnitus in their sample. Adolescents listening for $\geq 3$ hours are more prone to suffering from tinnitus. ${ }^{20,26,27-30}$ Prevalence of hearing problems, i.e., tinnitus and perceived hearing impairment were $6.1 \%$ and $5.8 \%$ respectively. Perceived hearing impairment and tinnitus were more seen in males than females. Women perceived they were more sensitive to noise then men. ${ }^{31}$ 


\section{Hearing thresholds of college-going students who use headphones for listening}

Out of the 341 respondents, 30 were taken up for hearing testing using pure tone audiometry. Two pure tone averages were obtained for each ear of the 30 participants - average of $500 \mathrm{~Hz}, 1000 \mathrm{~Hz}$ and $2000 \mathrm{~Hz}$ (PTA) and average of $4000 \mathrm{~Hz}, 6000 \mathrm{~Hz}, 8000 \mathrm{~Hz}$ and 10000 $\mathrm{Hz}$ (HFA PTA). Extended high-frequency audiometry is found to be a sensitive method for early detection of Noise Induced Hearing Loss as depicted in a study by Peng et al..$^{32}$ The 30 participants in the present study were divided into 2 groups, those who reported to perceive hearing difficulties and those who did not report to perceive hearing difficulties. It is seen from the results that for the group of participants who reported hearing difficulty, the audiometric testing indicated "pass" result for PTA left and HF PTA left while it indicated a refer result for PTA right and HF PTA right. Similarly, for the group of participants who reported no hearing difficulty, the audiometric results (PTA right, PTA left, HF PTA right, and HF PTA left) indicated a "refer" result. This difference was significant for HF PTA of left ear where the mean HF PTA for participants with no reported hearing difficulty was $18.65 \mathrm{dBHL}$ while that for the group with perceived hearing difficulty was $10.72 \mathrm{dBHL}$. This difference can be primarily attributed to one participant whose HF PTA in left ear was $77.5 \mathrm{dBHL}$. Results show that participants who perceive to have hearing problems have variable results on pure tone audiometry and those who do not even perceive any problems related to hearing may have poorer thresholds comparatively. This was in agreement with the study done by Jiang et al. ${ }^{33}$ where worse hearing thresholds were found in personal listening device users using audiometry, and significantly poor results in otoacoustic emission (OAE), even in the participants with selfreported 'normal hearing'. These results show how important it is to have a regular hearing check-up even when you don't perceive any hearing difficulty or else it might go unnoticed and may progress gradually. Other studies involving hearing threshold measurement using headphones have reported similar results. ${ }^{25,26,30,32-35}$

\section{Correlation between self-reported hearing status and average hearing thresholds}

One objective of the study was to correlate the self-reported hearing problems with the high frequency pure tone average of the same individuals. The Pearson correlation coefficient between the self-reported hearing problems and audiometric hearing thresholds was found to be $\mathrm{r}=0.2304$, which indicates that there is weak positive correlation between the self-reported hearing problems and audiometric hearing thresholds. This was in agreement with Jiang et al. ${ }^{33}$ in which he also showed poor hearing thresholds and poor OAE on sample using headphones even in those who self-reported to have normal hearing. Widen et al. ${ }^{26}$ showed opposite results in which more noise exposure and longer headphone usage resulted in poor hearing thresholds and more self-perceived hearing related problems. Similarly, Båsjö et al. ${ }^{30}$ showed that the children who reported to have tinnitus had significantly poorer hearing thresholds in both ears. Positive correlation was found between hearing threshold, years of usage and volume setting in the study by Kumar et al. ${ }^{35}$ which states that with $>5$ years of PLD use subjects showed more difference in their hearing thresholds in extended high frequency audiometry. The hearing thresholds were significantly elevated in those listening to PLDs at high volume settings as compared to normal group and low volume users.

\section{Conclusions}

It can be concluded that majority of teenage population listen to music at significant loud volumes and through insert earphones and are aware of the ill effect caused by it on the hearing mechanism but still are not well versed with the ways in which it could be prevented further. Also, there was no significant difference found between the pure tone averages of the two groups, i.e., one group who perceive to have hearing problems and other group which does not perceive to have hearing problems, only difference found was between the HFPTA of left ear between the groups. There was a weak correlation found between the self-reported hearing problems and audiometric hearing thresholds.

The study has few limitations. The sample size taken for into consideration for audiometry was small. Equality of gender of the participants was not controlled. Output levels of different music players were not measured and compared. Among the audiological test battery, Oto-acoustic emission test is more sensitive to the ill-effects of noise. However, the present study did not incorporate Oto-acoustic emission testing. This study was unable to educate the sample about the prevention methods to lessen the ill effect of excessive exposure to loud sounds.

\section{Acknowledgments}

The authors thank the Director and officials of AYJNISHD, Mumbai for permitting to conduct the study at the institute.

\section{Conflicts of interest}

The authors declare no conflict of interest.

\section{Funding}

None.

\section{References}

1. Moore BCJ. Cochlear Hearing Loss. London: Whurr Publishers, 1998.

2. Kujawa SG, Liberman MC. Acceleration of age-related hearing loss by early noise exposure: Evidence of a misspent youth. $J$ Neurosci. 2006;26(7):2115-2123

3. Arts HA. Sensorineural hearing loss in adults. In: Cummings CW \& Flint P Wet al., eds. Otolaryngology: Head \& Neck Surgery. 5 th ed. Philadelphia, PA: Elsevier Mosby; chap. 2010: 149.

4. South T. Managing Noise and vibration at work. Oxford; Elsevier Ltd. 2004:1-280.

5. Noise and Hearing Loss. The Journal of the American Medical Association. JAMA. 1990; 263(23):3185

6. Jokitulppo J, Björki E, Akaan-Penttiä E. Estimated Leisure Noise Exposure and Hearing Symptoms in Finnish Teenagers. International Journal of Audiology. 1997;26(4):257-262.

7. Henderson E, Testa M, Hartnick C. Prevalence of Noise-Induced HearingThreshold Shifts and Hearing Loss Among US Youths. PEDIATRICS 2010;127(1):e39-e46.

8. Lees RE, Roberts JH, Wald Z. Noise induced hearing loss and leisure activities of young people: a pilot study. Can J Public Health. 1985;76(3):171-173.

9. Blair JC, Hardegree D, Benson PV. Necessity and effectiveness of a hearing conservation program for elementary students. $J$ Educ Audiol. $1996 ; 4: 12-16$. 
10. World Health Organization. 1.1 billion People at risk of hearing loss.

11. Holgers K, Pettersson B. Noise exposure and subjective hearing symptoms among school children in Sweden. Noise and Health. 2005;7(27):27-37.

12. Zhao F, Manchaiah V, French D, et al. Music exposure and hearing disorders: An overview. International Journal of Audiology. 2009;49(1):54-64.

13. Kim M, Hong S, Shim H, et al. Hearing Threshold of Korean Adolescents Associated with the Use of Personal Music Players. Yonsei Medical Journal. 2009;50(6):771-776.

14. Vogel I, Verschuure H, van der Ploeg CP, et al. Adolescents and MP3 players: Too many risks, too few precautions. Pediatrics. 2009;123(6):e953-e958.

15. Kumar A, Mathew K, Alexander SA, et al. Output sound pressure levels of personal music systems and their effect on hearing. Noise Health. 2009;11(44):132-140.

16. Daniel E. Noise and hearing loss: a review. J Sch Health. 2007;77(5):225231.

17. Fligor B, Cox L. Output Levels of Commercially Available Portable Compact Disc Players and the Potential Risk to Hearing. Ear and Hearing. 2004;25(6):513-527.

18. Portnuff C, Fligor B, Arehart K. Teenage Use of Portable Listening Devices: A Hazard to Hearing? Journal of the American Academy of Audiology. 2011;22(10):663-677.

19. Portnuff C. Reducing the risk of music-induced hearing loss from overuse of portable listening devices: understanding the problems and establishing strategies for improving awareness in adolescents. Adolescent Health, Medicine and Therapeutics. 2016;7:27-35.

20. Gupta N, Sharma A, Singh P, et al. Assessment of Knowledge of Harmful Effects and Exposure to Recreational Music in College Students of Delhi: A Cross Sectional Exploratory Study. Indian Journal of Otolaryngology and Head \& Neck Surgery. 2013;66(3):254-259.

21. McNeill K, Keith S, Feder K, et al. MP3 player listening habits of 17 to 23 year old university students. The Journal of the Acoustical Society of America. 2010;128(2):646-653.

22. Torre P. Young Adults' Use and Output Level Settings of Personal Music Systems. Ear and Hearing. 2008;29(5):791-799.

23. Herrera S, Lacerda A, Lürdes D, et al. Amplified music with headphones and its implications on hearing health in teens. The International Tinnitus Journal. 2016;20(1):42-47.
24. Berg A, Ibrahim H, Sandler S, et al. Music-Induced Hearing Loss: What Do College Students Know? Contemporary Issues in Communication Science and Disorders. 2016;43(Fall):195-205.

25. Shah S, Gopal B, Reis J, et al. Hear today, gone tomorrow: An assessment of portable entertainment player use and hearing acuity in a community sample. J Am Board Fam Med. 2009;22(1):17-23.

26. Widen S, Båsjö S, Möller C, et al. Headphone listening habits and hearing thresholds in Swedish adolescents. Noise and Health. 2017;19(88):125132.

27. Kähäri K, Åslund T, Olsson J. Preferred sound levels of portable music players and listening habits among adults: A field study. Noise and Health. 2011;13(50):9-15.

28. Hodgetts W, Rieger J, Szarko R. The Effects of Listening Environment and Earphone Style on Preferred Listening Levels of Normal Hearing Adults Using an MP3 Player. Ear and Hearing. 2007;28(3):290-297.

29. Keppler H, Dhooge I, Vinck B. Hearing in young adults. Part II: The effects of recreational noise exposure. Noise and Health. 2015;17(78): 245-252.

30. Båsjö S, Möller C, Widén S, et al. Hearing thresholds, tinnitus, and headphone listening habits in nine-year-old children. International Journal of Audiology. 2016;55(10):587-596.

31. Bohlin M, Erlandsson S. Risk behaviour and noise exposure among adolescents. Noise and Health. 2007;9(36):55-63.

32. Peng J, Tao Z, Huang Z. Risk of Damage to Hearing from Personal Listening Devices in Young Adults. The Journal of Otolaryngology. 2007;36(03):181-185.

33. Jiang W, Zhao F, Guderley N, et al. Daily music exposure dose and hearing problems using personal listening devices in adolescents and young adults: A systematic review. International Journal of Audiology. 2016;55(4):197-205.

34. Mohammadpoorasl A, Hajizadeh M, Marin S, et al. Prevalence and Pattern of Using Headphones and Its Relationship with Hearing Loss among Students. Health Scope. 2018;7(4).

35. Kumar P, Upadhyay P, Kumar A, et al. Extended high frequency audiometry in users of personal listening devices. American Journal of Otolaryngology. 2017;38(2):163-167. 